\section{The capacity of Listeria monocytogenes mutants with in-frame deletions in putative ATP-binding cassette transporters to form biofilms and comparison with the wild type}

\author{
Marina Ceruso, ${ }^{1}$ Pina Fratamico, ${ }^{2}$ \\ Claudia Chirollo, ${ }^{1}$ Rosanna Taglialatela, ${ }^{1}$ \\ Maria Luisa Cortesi, ${ }^{1}$ Tiziana Pepe ${ }^{1}$ \\ 'Dipartimento di Medicina Veterinaria e \\ Produzioni animali, Università degli Studi \\ Federico II, Napoli, Italy; ${ }^{2}$ Molecular \\ Characterization of Foodborne Pathogens \\ (MCFP), Eastern Regional Research \\ Center (ERRC), United States \\ Department of Agriculture (USDA), \\ Wyndmoor, PA, USA
}

\section{Abstract}

Listeria monocytogenes $(\mathrm{Lm})$ is a food-borne pathogen responsible for human listeriosis, an invasive infection with high mortality rates. Lm has developed efficient strategies for survival under stress conditions such as starvation and wide variations in temperature, $\mathrm{pH}$, and osmolarity. Therefore, $\mathrm{Lm}$ can survive in food under multiple stress conditions. Detailed studies to determine the mode of action of this pathogen for survival under stress conditions are important to control $L m$ in food. It has been shown that genes encoding for ATP-binding cassette ( $\mathrm{ABC}$ ) transporters are induced in $\mathrm{Lm}$ in food, in particular under stress conditions. Previous studies showed that these genes are involved in sensitivity to nisin, acids, and salt. The aim of this study was to determine the involvement of some $\mathrm{ABC}$ transporters in biofilm formation. Therefore, deletion mutants of $\mathrm{ABC}$ transporter genes (LMOf2365_1875 and LMOf2365 1877) were created in $L m$ F2365, and then were compared to the wild type for their capacity to form biofilms. $\mathrm{Lm}$ strain F2365 was chosen as reference since the genome is fully sequenced and furthermore this strain is particularly involved in food-borne outbreaks of listeriosis. Our results showed that $4 L M O f 2365 \_1875$ had an increased capacity to form biofilms compared to the wild type, indicating that LMOf2365_1875 negatively regulates biofilm formation. A deeper knowledge on the ability to form biofilms in these mutants may help in the development of intervention strategies to control $\mathrm{Lm}$ in food and in the environment.

\section{Introduction}

Listeria monocytogenes $(\mathrm{Lm})$ can cause listeriosis, a severe invasive disease with high hospitalization ( $>90 \%)$ and mortality rates (20 to $30 \%$ ), especially in immunocompromised people, elderly individuals, and pregnant women. Therefore listeriosis is an infection of great concern to public health despite its low incidence ( 0.4 cases per 100,000 population) (EFSA, 2011). $\mathrm{Lm}$ is a food-borne pathogen of significant concern also to the food processing industry because of its ability to grow in food under multiple stress conditions (Nair et al., 2000). A better understanding of the mechanisms of $\mathrm{Lm}$ for survival under stress conditions is important to control this pathogen in food. In response to changes in the natural environment, bacteria undergo a complex program of differential gene expression. A number of transcriptional regulators important for stress response gene expression have been identified in Lm (Hanawa et al., 2000; Leimeister-Wachter et al., 1990; Nair et al., 2000). ATP-binding cassette (ABC) transporters genes have been shown to be induced in $\mathrm{Lm}$ subjected to high pressure and under stress conditions (Liu and Ream, 2008; Liu et al., 2012b). Previous studies showed that these genes are involved in sensitivity to nisin, acids, and salt (Liu et al., 2012a). All ABC transporters are either exporters or importers. There are more than 30 copies of different $A B C$ transporters in the genome of $\mathrm{Lm}$. Some ABC transporters have been shown to be involved in biofilm formation (Zhu et al., 2008, 2011; Vanderlinde et al., 2010; Seaton et al., 2011). The manganese $\mathrm{ABC}$ transporters LMOf2365_1875 and LMOf2365_1877 were highly induced in milk (Liu and Ream, 2008). Manganese is involved in a number of cellular functions such as virulence and oxidative stress (Papp-Wallace and Maguire, 2006). Since the ABC the transporter operon was induced with a number of treatments such as high pressure and nisin (Liu et al., 2011), it may be supposed that it is also involved in $\mathrm{Lm}$ ability to form biofilm. Therefore, the in-frame deletion mutants, $\triangle L M O f 236 \_1875$ and LMOf2365 1877 were constructed, and tested for their capacity to form biofilms in comparison with the wild type.

\section{Materials and Methods}

Lm strain F2365 isolated from Mexican-style soft cheese that had been implicated in an outbreak of listeriosis in California in 1985 (Linnan et al., 1988) was used in this study since its genome is fully sequenced and annotated (Nelson et al., 2004).
Correspondence: Tiziana Pepe, Dipartimento di Medicina Veterinaria e Produzioni animali, Università degli Studi Federico II, via Delpino 1, 80137 Napoli, Italy.

Tel. +39.081.2533905 - Fax: +39.081.292981.

E-mail: tiziana.pepe@unina.it

Key words: $\mathrm{ABC}$ transporters, Biofilm, L. monocytogenes.

Received for publication: 9 May 2013.

Revision received: 2 September 2013.

Accepted for publication: 2 September 2013.

This work is licensed under a Creative Commons Attribution 3.0 License (by-nc 3.0).

CCopyright M. Ceruso et al., 2014

Licensee PAGEPress, Italy

Italian Journal of Food Safety 2014; 3:1657

doi:10.4081/ijfs.2014.1657

The construction of in-frame deletion mutants $\triangle L M O f 2365 \_1875$ and LMOf2365_1877 in Lm F2365 was performed according Liu et al. (2012a). Glycerol stock cultures of $L m F 2365$ and isogenic mutants of this parent strain stored at $-80^{\circ} \mathrm{C}$ were streaked onto Brain Heart Infusion (BHI) (SigmaAldrich, St. Louis, MO, USA) agar plates and grown at $37^{\circ} \mathrm{C}$ prior to each experiment. Five milliliters of Mueller-Hinton broth overnight cultures for $\mathrm{Lm}$ strains $\mathrm{Lm} 2365, \mathrm{Lm} \mathrm{1875}$, and Lm 1877 were initiated from plate grown cultures. The overnight cultures were incubated at $32^{\circ} \mathrm{C}$ with agitation $(200 \mathrm{rpm})$, and the he next day, the overnight cultures were diluted 1:100 into fresh Mueller-Hinton broth. Flat bottom cell culture 96-well microtiter plates (Greiner Bio-one, Monroe, NC, USA) were washed with $100 \%$ Ethanol (ETOH) and allowed to air dry in a biological hood until all residual ETOH had evaporated. For each strain, $100 \mu \mathrm{L}$ of the freshly diluted culture were placed in 8 different wells. Additionally, as a negative control, 100 $\mu \mathrm{L}$ of sterile Mueller-Hinton broth were also placed into 8 additional wells. The plates were incubated statically at $32^{\circ} \mathrm{C}$ for $48 \mathrm{~h}$. The wells were then observed to see if visible biofilms were present in the $\mathrm{Lm}$ inoculated wells. The medium was then removed from the microtiter plate wells, and the individual wells were washed 5 times with $150 \mu \mathrm{L}$ of sterile distilled water. The plates were then allowed to air dry for $45 \mathrm{~min}$, and then $150 \mu \mathrm{L}$ of a $1 \%$ crystal violet solution were added to each of the wells. After $45 \mathrm{~min}$, the stain was removed and the wells were washed 5 times with $150 \mu \mathrm{L}$ sterile distilled water. The wells were then destained of the residual biofilm bound crystal violet with $200 \mu \mathrm{L}$ of $95 \%$ ETOH. One hundred microliters of the destain mixture were then transferred to a new microtiter plate, and the OD 590nm was measured for each well. The resulting data for 
be involved in the capacity of $L m$ to form biofilms. Since ATP-Binding Cassette transporters have been shown to be involved in nisin resistance and sensitivity to acids and salt, it may be hypothesized that these genes could be used as targets for the development of new antimicrobials in food, but not to prevent the biofilms formation. EC Regulation 2073/2005 on the microbiological criteria for foodstuffs, contains provisions for $\mathrm{Lm}$, and the competent authority has to verify compliance with the rules and criteria laid down in this Regulation. The results of this study, related to the molecular basis of virulence and stress responses of $\mathrm{Lm}$, may help in the development of targeted intervention strategies and treatments for the control of the pathogen in foods. ferences $(P<0.05)$ in the mean values generated by the $\mathrm{L}$. monocytogenes strains. Error bars represent the standard error of the means.

three separate trials was subjected to an analysis of variance. The individual trials were considered as a block when performing the mean separations using the least significant difference technique at a $\mathrm{P}<0.05$ level (Miller, 1981).

\section{Results}

Results demonstrated that LLMOf2365_1875 formed more biofilm than the wild type whereas bioflm formation by $\triangle L M O f 2365 \_1877$ was similar to the wild type (Figure 1).

\section{Discussion and Conclusions}

Functional genomics research on $\mathrm{Lm}$ allows a better understanding of the genes related to stress responses, and this knowledge may help in the development of intervention strategies to control this food-borne pathogen. In $L$. monocytogenes, one $\mathrm{ABC}$ transporter (lm.G_1771) encoding a putative $\mathrm{ABC}$ transporter permease has been identified to be involved in the negative regulation of biofilm formation since deletion of this gene resulted in increased capacity in biofilm formation (Zhu et al., 2008). In our study, LLMOf2365_1875 also showed increased capacity in biofilm formation compared to the wild type, although LMOf2365_1875 showed very little homology to $L m G \_1771$. These results suggest that these two genes may both
Microbiol 74:6859-66.

Liu Y, Ream A, Joerger RD, Liu J, Wang Y, 2011. Gene expression profiling of a pressuretolerant Listeria monocytogenes Scott A ctsR deletion mutant. $\mathrm{J}$ Ind Microbiol 38:1523-33.

Miller RG, 1981. Simultaneous statistical inference. 2nd ed. Springer-Verlag, New York, NY, USA.

Nair S, Derre I, Msadek T, Gaillot 0, Berche P, 2000. CtsR controls class III heat shock gene expression in the human pathogen Listeria monocytogenes. Mol Microbiol 35:800-11.

Nelson KE, Fouts DE, Mongodin EF, Ravel J, DeBoy RT, Kolonay JF, Rasko DA, Angiuoli SV, Gill SR, Paulsen IT, Peterson J, White O, Nelson WC, Nierman W, Beanan MJ, Brinkac LM, Daugherty SC, Dodson RJ, Durkin AS, Madupu R, Haft DH, Selengut J, Van Aken S, Khouri H, Fedorova N, Forberger H, Tran B, Kathariou S, Wonderling LD, Uhlich GA, Bayles D0, Luchansky JB, Fraser CM, 2004. Whole genome comparisons of serotype $4 \mathrm{~b}$ and $1 / 2 \mathrm{a}$ strains of the food-borne pathogen Listeria monocytogenes reveal new insights into the core genome components of this species. Nucleic Acids Res 32:238695.

Papp-Wallace KM, Maguire ME, 2006. Manganese transport and the role of manganese in virulence. Annu Rev Microbiol 60:187-209.

Seaton K, Ahn SJ, Sagstetter AM, Burne RA, 2011. A transcriptional regulator and $A B C$ transporters link stress tolerance, (p)ppGpp, and genetic competence in Streptococcus mutans. J Bacteriol. 193:862-74.

Vanderlinde EM, Harrison JJ, Muszyński A, Carlson RW, Turner RJ, Yost CK, 2010. Identification of a novel $\mathrm{ABC}$ transporter required for desiccation tolerance, and biofilm formation in Rhizobium leguminosarum bv. viciae 3841. FEMS Microbiol Ecol 71:327-40.

Zhu X, Liu W, Lametsch R, Aarestrup F, Shi C, She Q, Shi X, Knøchel S, 2011. Phenotypic, proteomic, and genomic characterization of a putative $\mathrm{ABC}$-transporter permease involved in Listeria monocytogenes biofilm formation. Foodborne Pathog Dis 8:495-501.

Zhu X, Long F, Chen Y, Knøchel S, She Q, Shi X, 2008. A putative $\mathrm{ABC}$ transporter is involved in negative regulation of biofilm formation by Listeria monocytogenes. Appl Environ Microb 74:7675-83. 